$41.8 \%$ of examinations were normal. The commonest finding in patients with IDA was angioectasia (19.9\%). $59.5 \%$ of patients with obscure overt GI blood loss had positive findings. (Active bleeding (24.3\%), Vascular lesion (5.4\%), polyp (10.8\%), NSAID enteropathy $(2.7 \%)$, and gastritis $(2.7 \%))$. Crohn's disease was identified in $5.8 \%$ of all cases and in $13.8 \%$ of cases referred for assessment of suspected Crohn's disease. $4.6 \%$ of patients with IDA had findings consistent with Crohn's disease.

Further investigation was advised in $36.7 \%$. In $35.9 \%$ of those patients, the major abnormality identified for which a further procedure was required was located within the reach of a gastroscope. In those with IDA, gastroscopy was recommended in $22.7 \%$ of cases. Gastroscopy was recommended in a smaller proportion of patients (4.4\%) having CE for other indications.

A repeat procedure with bowel cleansing agents was required because of poor views in $1.6 \%$ of procedures.

Capsule retention occurred in 2 of 186 patients (1.1\%) for whom retention data was available. Prior small bowel imaging had not demonstrated a stricture in either case. Endoscopic removal of the capsule was successful in both patients. The cause of capsule retention was crohn's disease in one and NSAID enteropathy in the other.

Conclusion Adequate diagnostic images were obtained in $>98 \%$ of patients without the administration of bowel cleansing agents. The yield of positive findings from capsule endoscopy is high, but a significant proportion of lesions are within the range of a gastroscope, especially in patients with IDA. We recommend that patients undergo at least two gastroscopies prior to CE. CE is a safe procedure. Capsule retention was reported with a similar frequency to that reported in other case series. Patients should be advised that normal small bowel radiology may not exclude a significant stricture. Disclosure of Interest None Declared

\section{PTU-044 JOINT ENDOSCOPIC/LAPAROSCOPIC PROCEDURES FOR MANAGEMENT OF COMPLEX COLONIC POLYPS}

doi:10.1136/gutjnl-2013-304907.136

1,"E Wesley, ${ }^{2} \mathrm{~N}$ L Bullen, ${ }^{2} \mathrm{~N}$ J Smart, ${ }^{2} \mathrm{~S} D$ Mansfield, 'T Shirazi. 'Gastroenterology; ${ }^{2}$ Surgery, Royal Devon and Exeter Hospital, Exeter, UK

Introduction Polypectomy during colonoscopy can be challenging and is potentially dangerous. Patients with particularly challenging polyps have been traditionally referred for segmental colectomy.

Laparo-endoscopic resection (LER) has been found to be safe and effective in other centres ${ }^{1,2}$. Laparascopic mobilisation of the colon for endoscopic sub-mucosal resection (EMR) has the potential to assist with the removal of complex polyps and can allow immediate management of complications.

We implemented a pathway for patients who would previously have been offered segmental resection to undergo colonoscopy in theatre under general anaesthetic (GA) by an expert colonoscopist with a laparoscopic colorectal surgeon present to assist with laparoscopic mobilisation or proceed to segmental colectomy if required.

Our aim was to assess the safety and feasibility of this new service and to compare our results to published data.

Methods Cases were collected prospectively from February 2010 to September 2012.

Data on patient demographics, indication, lesion site and size, index colonoscopist, LER surgeon and endoscopist, procedural details, length of hospital stay, completeness of endoscopic resection and complications were collected retrospectively by two independent investigators.

Results 25 patients, (17 male) underwent GA colonoscopy in theatre for 26 polyps. Polyp size estimation at initial colonoscopy ranged from $10-50 \mathrm{~mm}$, median $25 \mathrm{~mm}$. 15 polyps were in the right colon.
$8(32 \%)$ required segmental colectomy: 5 lesions appeared malignant, 1 was too large, 1 and 2 technically impossible to resect. 2 cases required laparoscopic mobilisation of the colon to aid EMR.

15 patients (60\%) avoided any abdominal surgical intervention.

Median length of hospital stay was 1 day.

2 patients had significant post-operative bleeding (1 EMR, 1 port-site). 1 patient developed a collection after laparoscopic resection which required radiological drainage. 1 EMR patient had polyp cancer with inadequate resection margins and required subsequent laparoscopic resection.

Conclusion Although the number of patients is relatively small, the pathway is a safe and feasible way to reduce the need for colonic resection for complex polyps. A significant number of patients avoided the need for segmental resection, and most did not require any laparoscopic assistance.

Disclosure of Interest None Declared

\section{REFERENCES}

1. Franklin, M.E., Jr., et al. Laparoscopically monitored colonoscopic polypectomy: an established form of endoluminal therapy for colorectal polyps. Surgical endoscopy 21, 1650-1653 (2007).

2. Wood, J.J., Lord, A.C., Wheeler, J.M. \& Borley, N.R. Laparo-endoscopic resection for extensive and inaccessible colorectal polyps: a feasible and safe procedure. Annals of the Royal College of Surgeons of England 93, 241-245 (2011).

\section{PTU-045 DIAGNOSTIC UTILITY OF SINGLE-USER PERORAL CHOLANGIOSCOPY FOR INDETERMINATE BILIARY STRICTURES IN SCLEROSING CHOLANGITIS}

doi:10.1136/gutjnl-2013-304907.137

1,"E Kalaitzakis, ${ }^{2} \mathrm{R}$ Sturgess, ${ }^{2,3} \mathrm{H}$ Kaltsidis, ${ }^{4} \mathrm{~K}$ Oppong, ${ }^{2} \mathrm{~V}$ Lekharaju, ${ }^{1 \mathrm{P}}$ Bergenzaun, ${ }^{3 P}$ Vlavianos, ${ }^{5} \mathrm{H}$ Sharma, ${ }^{3} \mathrm{D}$ Westaby, ${ }^{5} \mathrm{G} J$ Webster. ${ }^{1}$ Department of Gastroenterology, Skåne University Hospital, Lund, Sweden; '2Department of Gastroenterology, Aintree University Hospital, Liverpool; 'BDepartment of Gastroenterology, Imperial College Healthcare NHS Trust, London; 'Department of Gastroenterology, Freeman Hospital, Newcastle, ${ }^{5}$ Department of Gastroenterology, University College Hospital, London, UK

Introduction Single-user peroral cholangioscopy (SOC) has been reported to be useful in the differential diagnosis of indeterminate biliary lesions, but its diagnostic utility in sclerosing cholangitis(SC) has not been evaluated.

Methods Fifty-one consecutive patients with sclerosing cholangitis(47 primary SC; 4 IgG4-related cholangitis), who underwent 54 attempted SOC procedures due to indeterminate biliary strictures, in 4 UK and 1 Swedish tertiary referral centres in 2008-2012, were retrospectively enrolled. Brush cytology or SOC-directed biopsies were obtained as clinically indicated. The operating characteristics of SOC for detecting cancer were calculated using a composite reference standard (incl. surgical confirmation, tissue sampling, follow-up). For each SOC procedure in SC, another one attempted due to an indeterminate biliary stricture in patients without SC in the same centre and calendar year was randomly selected as control.

Results The Spyglass ${ }^{\mathrm{TM}}$ cholangioscope could be successfully inserted in the bile duct less frequently in SC compared to controls $(83 \%$ vs $96 \%, p=0.05)$, but the two groups did not differ in the mucosal visualisation success rate( $93 \%$ vs $94 \%)$. SOC-directed biopsies were more commonly performed in SC vs controls (83\% vs $61 \%, p=0.02)$ but the rate of adequate samples did not differ significantly ( $67 \%$ vs $77 \%, p=0.2)$. The sensitivity, specificity and accuracy of SOC with/without tissue sampling for cancer diagnosis were similar in the 2 groups(intention-to-diagnose; table). The SOC diagnosis rate of malignant lesions was also similar in SC vs controls ( $50 \%$ vs $55 \%, p>0.05)$. Adverse events were more common in SC, mainly due to an increased frequency of cholangitis (table). No severe adverse events were observed. 


\section{Abstract PTU-045 Table}

\begin{tabular}{lll}
\hline & Sclerosing cholangitis (n= 54) & Controls (n= 54) \\
\hline Age (yr) & $53.5(27)^{*}$ & $61(15)^{*}$ \\
Male gender & $72 \%^{*}$ & $52 \%^{*}$ \\
Follow-up (mo) & $9.5(13)$ & $10(9)$ \\
General anaesthesia & $56 \%$ & $47 \%$ \\
Sensitivity & $50 \%(35 \%>65 \%)$ & $55 \%(41-69 \%)$ \\
Specificity & $100 \%(100 \%>100 \%$ & $97 \%(91 \%>100 \%)$ \\
Accuracy & $88 \%(79 \%>98 \%)$ & $80 \%(68 \%>91 \%)$ \\
Adverse events & $17.5 \%$ & $7.5 \%$ \\
Cholangitis & $11 \%^{*}$ & $1.9 \% *$ \\
\hline
\end{tabular}

Data are presented as medians (IOR) and percentage as appropriate. The $95 \%$ confidence intervals of operating characteristics are reported. ${ }^{*} \mathrm{p} \leq 0.05$.

Conclusion In experienced hands, SOC is equally accurate in cancer diagnosis in SC and patients with single biliary strictures. SOC may be of particular use in the assessment of SC, in which differentiating benign from malignant strictures remains particularly challenging. However, cholangioscope insertion may be hampered by bile duct narrowing and cholangitis is more common following SOC in SC.

Disclosure of Interest None Declared

\section{PTU-046 A RETROSPECTIVE COMPARISON OF PLASTIC, UNCOVERED AND FULLY COVERED METAL STENTS IN THE MANAGEMENT OF DISTAL MALIGNANT BILIARY STRICTURES}

doi:10.1136/gutjnl-2013-304907.138

1."F Sampaziotis, '1 Evans, 'A King, 'B Macfarlane, 'A Leahy. 'Gastroenterology, Watford General Hospital, Watford, UK

Introduction Uncovered self expanding metal stents (USEMS) are associated with longer patency and reduced complication rates compared to polyethylene stents (PS), for the management of distal malignant biliary strictures (DMBS) [1]. However, PS are used for unstaged tumours, due to difficulties in operative USEMS removal. Fully covered self expanding metal stents (FCSEMS) can be easily removed before or during surgical resection. Recent data supports their use as first line management of DMBS, regardless of resectability status [2]. The current study provides the first retrospective comparison of survival, patency and complication rates between PS, USEMS and FCSEMS in the context of DMBS

Methods The records of all patients, who underwent endoscopic retrograde cholangiopancreatography and stent placement for newly diagnosed DMBS in our centre, between February 2007 and August 2012 were reviewed. Patients who subsequently underwent surgical resection were excluded from the patient survival estimation. The stent patency period was calculated as the period between stent insertion and occlusion; death or surgical resection with a patent stent. Cumulative patient survival and stent patency were estimated using the Kaplan Meier method. The log-rank test was used for comparison between groups. Fisher's exact test was used for comparison of complication rates. The statistical analysis software used was IBM SPSS Statistics 20.

Results 87 cases were identified: 37 PS (8.5 or 10 Ff), 26 USEMS (wallstent/wallflex) and 24 FCSEMS (wallflex). 7 patients proceeded to surgical resection. Stent occlusion occurred in 54\%, $23 \%$ and $4.2 \%$ of the patients in the PS, USEMS and FCSEMS group respectively, with a mean patency period of 87, 144 and 196 days. Cumulative patency was higher in the FCSEMS vs USEMS group $(p=0.028)$ and higher in both groups compared to the PS group $(p<0.001$ and $p=0.007$ respectively) [Fig1]. No statistically signifi- cant difference was observed in survival or complications between the 3 groups. However, 6 episodes of cholangitis occured in the PS group vs 1 episode in each of the FCSEMS and USEMS groups; 2 episodes of pancreatitis were noted in the FCSEMS group vs none in the other groups.

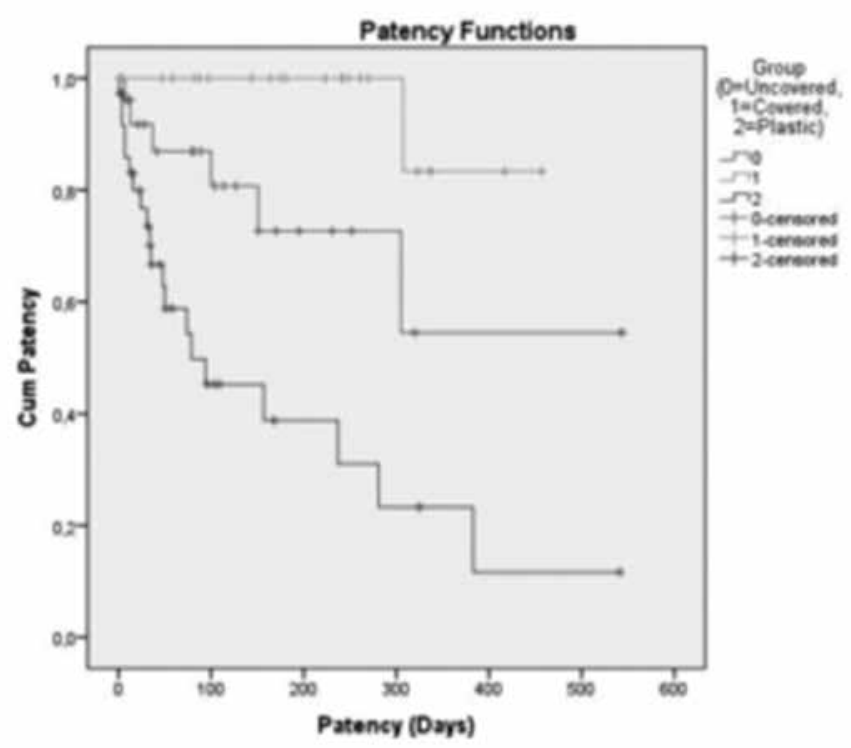

Abstract PTU-046 Figure

Conclusion The use of FCSEMS as first line management for DMBS is associated with longer patency and similar complication rates compared to USEMS and PS. However, the risk of pancreatitis requires further assessment.

Disclosure of Interest None Declared

\section{REFERENCES}

1. Lammer J, et al. Common bile duct obstruction due to malignancy: Treatment with plastic versus metal stents. Radiology 1996; 201:167-72.

2. Siddiqui AA, et al. Fully covered self-expandable metal stents are effective and safe to treat distal malignant biliary strictures, irrespective of surgical resectability status. J Clin Gastroenterol. 2011; 45:824-7.

\section{PTU-047 MANAGEMENT OF POLYP CANCERS WITHIN THE BOWEL CANCER SCREENING PROGRAMME COULD BE IMPROVED!}

doi:10.1136/gutjnl-2013-304907.139

1,*G Longcroft-Wheaton, 'P Bhandari. 'Gastroenterology, Portsmouth Hospitals NHS Trust, Portsmouth, UK

Introduction Endoscopic management of early colonic neoplasia or polyp cancer remains unclear. There are no national guidelines or good quality data to guide clinicians with these difficult lesions. The aim of this study is to identify the incidence of early cancer and variation in practise of managing these lesions

Methods Data was prospectively collected in all patients undergoing screening within the Solent BCSP. The database was interrogated to identify all neoplasia. The endoscopists suspicion of cancer was noted from the reports. MDT outcome was recorded, along with final management of the cancer

Results In total 3976 patients underwent screening colonoscopy between $2007-2012$. N = 5768 neoplastic polyps found giving a mean polyp detection rate of $1.5 /$ patient. Cancer was found in $235 / 3976(6 \%)$ patients. Mean age was 67yrs. 142/235 (60\%) were male. 145/235 (62\%) had advanced cancer, confirmed at surgery. 